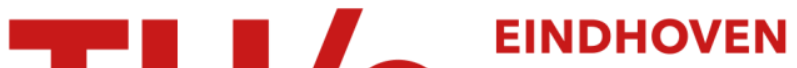 UNIVERSITY OF TECHNOLOGY
}

\section{Influence of particle shape in the additive manufacturing process for ceramics}

\section{Citation for published version (APA):}

Westbeek, S., van Dommelen, J. A. W., Remmers, J. J. C., \& Geers, M. G. D. (2019). Influence of particle shape in the additive manufacturing process for ceramics. Computers and Mathematics with Applications, 78(7), 23602376. https://doi.org/10.1016/j.camwa.2018.08.033

\section{Document license: \\ TAVERNE}

DOI:

10.1016/j.camwa.2018.08.033

Document status and date:

Published: 01/10/2019

\section{Document Version:}

Publisher's PDF, also known as Version of Record (includes final page, issue and volume numbers)

\section{Please check the document version of this publication:}

- A submitted manuscript is the version of the article upon submission and before peer-review. There can be important differences between the submitted version and the official published version of record. People interested in the research are advised to contact the author for the final version of the publication, or visit the $\mathrm{DOI}$ to the publisher's website.

- The final author version and the galley proof are versions of the publication after peer review.

- The final published version features the final layout of the paper including the volume, issue and page numbers.

Link to publication

\section{General rights}

Copyright and moral rights for the publications made accessible in the public portal are retained by the authors and/or other copyright owners and it is a condition of accessing publications that users recognise and abide by the legal requirements associated with these rights.

- Users may download and print one copy of any publication from the public portal for the purpose of private study or research.

- You may not further distribute the material or use it for any profit-making activity or commercial gain

- You may freely distribute the URL identifying the publication in the public portal.

If the publication is distributed under the terms of Article 25fa of the Dutch Copyright Act, indicated by the "Taverne" license above, please follow below link for the End User Agreement:

www.tue.nl/taverne

Take down policy

If you believe that this document breaches copyright please contact us at:

openaccess@tue.nl

providing details and we will investigate your claim. 


\title{
Influence of particle shape in the additive manufacturing process for ceramics
}

\author{
S. Westbeek ${ }^{\text {a,b }}$, J.A.W. van Dommelen ${ }^{\text {a,* }}$, J.J.C. Remmers ${ }^{\text {a }}$, M.G.D. Geers ${ }^{\text {a }}$ \\ ${ }^{a}$ Eindhoven University of Technology, Department of Mechanical Engineering, P.O. Box 513, 5600 MB Eindhoven, The Netherlands \\ ${ }^{\mathrm{b}}$ AMSYSTEMS Center, De Lismortel 31, 5612 AR Eindhoven, The Netherlands
}

\section{H I G H L I G H T S}

- Light propagation through the filled resin is studied for the AM process of ceramics.

- Maxwell's equations are incorporated to capture relevant wave-like behavior of light.

- A parametrized and systematic study investigates the influence of particle shape.

- The predictive value of a 2D simulation for a 3D setting is shown.

- The importance of accounting for multiple-scattering approaches is illustrated.

\section{A R T I C L E I N F O}

\section{Article history:}

Received 14 March 2018

Accepted 14 August 2018

Available online 1 September 2018

\section{Keywords:}

Additive manufacturing

Ceramics

Vat photopolymerization

Light modeling

Electromagnetics

Micro-scale modeling

\begin{abstract}
A B S T R A C T
Additive manufacturing (AM) of ceramics through a vat photopolymerization (VP) process is a promising technique due to the high intrinsic resolution and the evanescence of stresses introduced during the layerwise additive manufacturing process. Compared to the regular vat photopolymerization process, the addition of ceramic powder increases physical complexity. Hence, large scale adoption and optimization of AM for ceramics requires a thorough understanding of the underlying physics. This work focuses on the light scattering introduced through a complex interplay between optical properties of different constituents and its effect on the photopolymerization. The effect of the shape of the ceramic particle is investigated for isolated and multiple particle cases using a parametric analysis with regular convex polygons. Although the analysis of two-dimensional isolated particle shapes indicates a preference for one shape over the other, this difference vanishes in a multiple-scattering situation. A three-dimensional analysis furthermore shows that a two-dimensional approximation of the three-dimensional scattering problem is relevant and can be used to further the process understanding of ceramic VP.
\end{abstract}

(c) 2018 Elsevier Ltd. All rights reserved.

\section{Introduction}

Fabricating ceramics through additive manufacturing (AM) techniques is promising, facilitating near-net product fabrication that would not be feasible using conventional production techniques. Although it is possible to produce monolithic ceramics through a single-step process, the most prevailing and prosperous strategies fall into the multiple-step category [1-3].

\footnotetext{
* Corresponding author.

E-mail address: j.a.w.v.dommelen@tue.nl (J.A.W.van Dommelen).
} 


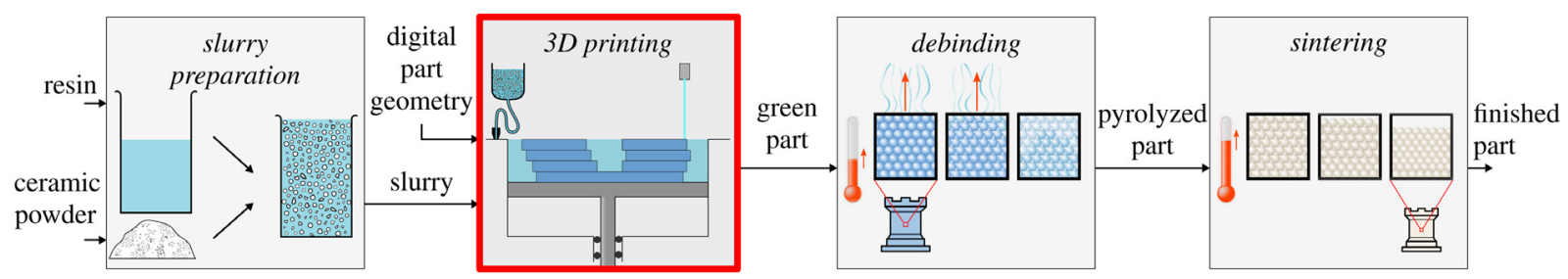

Fig. 1. Overview of the ceramic additive manufacturing process by vat photopolymerization.

The multi-step AM production approach considered here is based on vat photopolymerization (VP) and is shown in Fig. 1. Initially, ceramic powder with a particle diameter of the order of $1 \mu \mathrm{m}$ is dispersed into a photopolymeric resin with a high filling fraction, typically of the order of 50-60 vol\%. The resulting highly viscous fluid, termed a slurry, is then the base material for the indirect VP process. An intermediate "green" part is produced by a repetitive process of depositing a layer of slurry, patterned irradiation by UV light with a wavelength in the order of $\mathcal{O}(400 \mathrm{~nm})$ and lowering the base platform [4]. The green part, a composite material of ceramic inclusions in a polymer matrix is then subjected to a series of heat treatments, which can be subdivided into a debinding step where the polymer is evaporated and a subsequent sintering step where the part is sintered into a dense monolithic part [5]. Note that the concept of polymer derived ceramics is not new and the AM step can be replaced by other conventional polymer-forming techniques, such as injection molding [6]. Consequently, extensive knowledge and experience exists on the polymer-to-ceramic conversion [1,6]. The real interest therefore lies in the part shaping step by AM, i.e. the highlighted block in Fig. 1.

With the maturing of AM techniques in general, the need for suitable simulation tools to improve fundamental understanding of the process increases. A clear motivation for this can be found in the most prominent factors limiting a wide adoption of AM in industry, namely reproducibility, productivity and scalability [7]. For ceramics in particular, a number of additional challenges consist in increasing the material density, increasing feasible product sizes and wall thicknesses and avoiding the formation of cracks [1].

Another challenge in VP for ceramics is the light scattering introduced by the dispersed inclusions. This phenomenon is dominated by the difference in refractive indices between the resin and the ceramic particles [5,8-11]. As a result, the cured polymer geometry can be considerably different from the expected geometry for unfilled solutions (as a function of the light source). Additionally, the light dispersion through the slurry is also much less homogeneous due to an interplay between diffraction and interference at the considered length scales [12]. The inhomogeneous intensity distribution might, in turn, lead to an inhomogeneous polymer conversion or even the presence of residual monomer. The latter has been identified as a cause for crack formation during post-processing of the green part [13]. From the polymer-to-ceramic production chain it is known that high-quality green parts require a powder compact with a highly homogeneous microstructure and powder packing density [1]. The influence of powder shape is however less studied and clear guidelines are missing. In view of the light scattering phenomena, the actual shape might play an important role in reaching a homogeneous as possible final conversion. This work therefore aims to identify the influence of the particle shape in the vat photopolymerization step in the AM process.

Within the field of VP for ceramics, the primary interest in experimental and computational analysis has been focused on estimating the slurry's cure depth. Different factors have been recognized which influence the result, including the difference between refractive indices of the resin and powder, the powder volume fraction and (mean) diameter, and the light wavelength $[5,10,14,15]$. Typically, this dependence was found empirically. Similar approaches also exist to relate the cure depth to the cure width [10,14]. Many analytical approaches, such as the Kubelka-Munk theory, are limited to a twoway prediction, i.e. transmission and reflection in the opposite direction [16-18]. In order to numerically predict the full cure profile as a result of the applied light source, Sun \& Zhang $[19,20]$ implemented a Monte-Carlo ray tracing approach using Mie single-scattering theory to determine the probability density function for the scattering direction. An early warning that using a spherical shape (through e.g. Mie theory) for non-spherical particles may lead to ambiguous results was made by Thiele \& French [21]. They concluded this on the basis of a finite element scattering analysis of one (or two) representative rutile titania particle(s) in a coating.

In this work, a finite element model is used to obtain the near-field intensity field induced by the particle's shape, governing photopolymerization. In the following section the theory underlying this approach is addressed and two parameters are introduced to quantify the effect of the particle shape on the homogeneity of the intensity distribution and the resulting photopolymerization. The influence of particle geometry and orientation is first investigated in a two-dimensional setting in Section 3, using academic model problems with parametrized regular and convex polygon shapes. The translation to three-dimensions is made in Section 4, on platonic solids and shapes introduced to investigate the representative nature of a two-dimensional simulation in order to address a realistic 3D configuration. 


\section{Mathematical framework}

To identify the relevant phenomena induced by the particular shape of the inclusions on the electromagnetics fields, Maxwell equations are solved capturing the wave-like behavior of light. The reasoning for this is twofold. Firstly, due to the large filling fraction and the small difference between light wavelength and particle dimensions, which are both of the order of $1 \mu \mathrm{m}$, a wave description is required [22,23]. Phenomena such as interference and diffraction play an important role in this regime, where the ratio of the feature size to wavelength is smaller than ten. Refractive optics such as ray tracing are not able to capture these phenomena and should be applied only when the scattering object is over 100 times the size of the wavelength [22]. Secondly, the large filling fraction introduces so-called multiple-scattering that prohibits the application of single-scattering approaches such as the Mie theory, which is only applicable for highly dilute suspensions [23,24]. The theory described next is used to analyze the shape influence on the intensity homogeneity using both single- and multipleparticle and both 2D and 3D models in Sections 3 and 4.

\subsection{Wave equation}

In a numerical solution framework, the Maxwell equations are typically combined into a single formula. To this end, constitutive equations are used to eliminate the dependence of the electric field on the magnetic field or reversely. Here the former is used, such that the equilibrium equation reads [25]

$$
\vec{\nabla} \times\left(\frac{1}{\mu} \vec{\nabla} \times \vec{E}\right)+\epsilon \frac{\partial^{2} \vec{E}}{\partial t^{2}}+\sigma \frac{\partial \vec{E}}{\partial t}=-\frac{\partial \vec{J}^{\mathrm{imp}}}{\partial t},
$$

where $\vec{E}$ is the electric field, $\mu$ the magnetic permeability, $\epsilon$ the electrical permittivity, $\sigma$ the electrical conductivity, $\vec{\nabla} \times$ the curl-operator, $\vec{J}^{\text {imp }}$ the imposed electrical current density and $t$ the time. By definition, the total current density $\vec{J}$ is equal to $\vec{J}^{\mathrm{imp}}+\sigma \vec{E}$. Alternatively, under the assumption that the electric field takes the time harmonic form $\vec{E}(\vec{x}, t)=\Re\left(\vec{E}(\vec{x}) e^{i \omega t}\right)$, Eq. (1) can be rewritten in the frequency domain yielding $[25,26]$

$$
\vec{\nabla} \times\left(\frac{1}{\mu} \vec{\nabla} \times \vec{E}\right)-\left(\omega^{2} \epsilon-i \omega \sigma\right) \vec{E}=-i \omega \vec{J}^{\mathrm{imp}},
$$

where $\omega$ is the angular frequency. The analogy to light modeling becomes apparent by introducing the wave number $k$ in free space as $[26,27]$

$$
k_{0}=\frac{\omega}{c_{0}}=\omega \sqrt{\mu_{0} \epsilon_{0}},
$$

with $c_{0}$ the speed of light in vacuum and $\mu_{0}$ and $\epsilon_{0}$ the permeability and permittivity in vacuum. Considering the values in free space, $\mu_{0}$ and $\epsilon_{0}$, are constant, a relative dimensionless material parameter is often introduced in the form of $\mu_{r}$ and $\epsilon_{r}$, with $\mu=\mu_{0} \mu_{r}$ and $\epsilon=\epsilon_{0} \epsilon_{r}$. This, together with Eq. (3), allows rewriting Eq. (2) as

$$
\vec{\nabla} \times\left(\frac{1}{\mu_{r}} \vec{\nabla} \times \vec{E}\right)-k_{0}^{2}\left(\epsilon_{r}-\frac{i \sigma}{\epsilon_{0} \omega}\right) \vec{E}=\overrightarrow{0} .
$$

The second term is often simplified further using a complex form of the permittivity, i.e.

$$
\tilde{\epsilon_{r}}=\epsilon_{r}-\frac{i \sigma}{\epsilon_{0} \omega} \text {. }
$$

Since ceramics and polymers are typically dielectric, the relative magnetic permeability $\mu_{r}$ is (very close to) unity [27]. Consequently, the complex relative permittivity can be written as

$$
\tilde{\epsilon_{r}}=(n-i \kappa)^{2} \equiv \tilde{n}^{2},
$$

where $n$ is the refractive index and $\kappa$ the extinction coefficient of the material. Using $\mu_{r}=1$, the inhomogeneous vector curl-curl equation becomes [27]

$$
\vec{\nabla} \times(\vec{\nabla} \times \vec{E})=k_{0}^{2} \tilde{n}^{2} \vec{E}
$$

This differential equation is solved using the finite element framework. Note that the magnetic field $\vec{H}$ can be recovered through the original Maxwell's equations, i.e. through Faraday's law as $[25,27,28]$

$$
\vec{H}=-\frac{i}{\omega \mu} \vec{\nabla} \times \vec{E}
$$




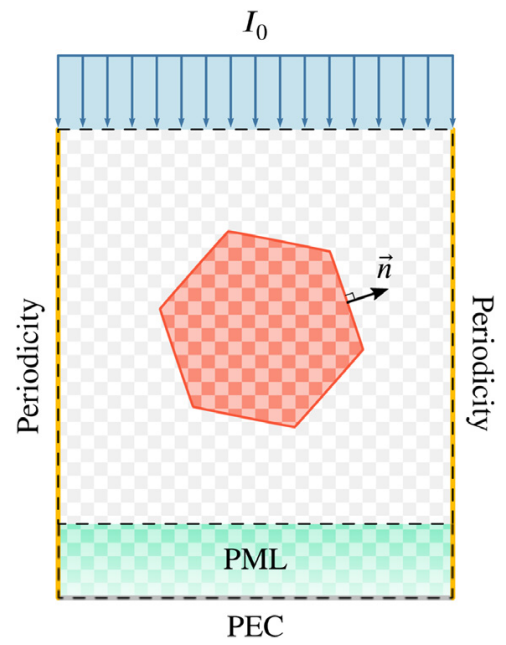

Fig. 2. Illustration of the considered boundary conditions.

\subsection{Boundary conditions}

The vector wave equation for the electric field must be complemented by appropriate boundary conditions. A visualization of the applied conditions is provided in Fig. 2.

Along the interface between different media, i.e. the matrix and inclusion material in this case as illustrated by the red line in Fig. 2, the jump conditions must be satisfied [28,29]:

$$
\begin{aligned}
& \vec{n} \times[\vec{E}]=\overrightarrow{0}, \\
& \vec{n} \cdot[(i \omega \epsilon+\sigma) \vec{E}]=0,
\end{aligned}
$$

where $[\cdot]$ denotes the jump of the quantities from one side of the interface to the other and $\vec{n}$ is the unit normal outward vector. A Neumann boundary acting as the port condition for the incoming light is provided as

$$
\vec{n} \times\left(\frac{1}{\mu} \vec{\nabla} \times \vec{E}\right)=-i \omega \vec{J}_{s}^{\mathrm{imp}},
$$

with $\vec{J}_{s}^{\mathrm{imp}}$ an imposed electrical surface current tangent to the boundary. The corresponding source intensity $I_{0}$, which will be defined in Section 2.4, is illustrated by the blue arrows in Fig. 2. In order to truncate the computational domain, an absorbing boundary condition is used in the form of a perfectly matched layer (PML) [30]. A PML can be considered as a finite layer surrounding the physical domain where the principle of coordinate stretching is applied through modification of the material parameters [28,31]. Typically, the domain extended by the PML is bounded by a perfect electric conductor (PEC) or Dirichlet boundary condition, which reads

$$
\vec{n} \times \vec{E}=\overrightarrow{0} .
$$

Alternatively, the considered domain size is also reduced by using periodic boundary conditions as indicated in Fig. 2. Within electromagnetics the corresponding boundary condition stems from Bloch-Floquet's theorem, which states that fields on opposite surfaces are related through a constant phase shift [28,32].

\subsection{Discretization}

Using regular FE elements in inhomogeneous electromagnetic problems can lead to non-physical or spurious solutions, primarily due to the lack of enforcement of the divergence condition [28]. Furthermore, imposing boundary conditions is inconvenient at material interfaces and conducting surfaces. Lastly, treating conducting and dielectric edges and corners is cumbersome due to the associated singularities. A common solution to these problems was proposed in the 1980s in the form of vector elements that assign degrees of freedom to the edges rather than to the nodes of the elements, thus called edge, vector or Nédélec elements [28,33-35]. The degrees of freedom of these edge elements typically correspond to the integral of the tangential component (of either $\vec{E}$ or $\vec{H}$ ) along the edges of the patches. Because a quadrilateral mesh shows a larger cumulative phase error in comparison to a triangular mesh, the latter will be used in the following [28]. In this work the finite element package Comsol 5.0 [26] is used for the corresponding numerical analyses. 


\subsection{Photopolymerization}

Although the numerical framework solves for the electric and magnetic fields, the real interest here lies in the (resulting) photopolymerization. In order to relate the electromagnetic waves to the chemical reaction, a conversion to light intensity/irradiance is required. The electric and magnetic fields are akin to the local intensity through the Poynting vector:

$$
\vec{S}=\vec{E} \times \vec{H}^{*},
$$

where $\vec{H}^{*}$ denotes the complex conjugate of the magnetic field $\vec{H}$ and $\vec{S}$ represents the directional energy flux in w/me intensity then simply follows from the magnitude of the time averaged Poynting vector $[26,27,36]$, i.e.

$$
I=\|\langle\vec{S}\rangle\|=\left\|\frac{1}{2} \Re\left\{\vec{E} \times \vec{H}^{*}\right\}\right\|,
$$

where $\langle\cdot\rangle$ indicates a time average and $\|\cdot\|$ the $L^{2}$-norm. For heterogeneous media, Eq. (13) provides the near-field information required to describe the polymerization process.

In order to quantify the influence of the ceramic powder, one option is to compare the local resulting intensity to the expected intensity without the presence of inclusions. If the magnitude of the absorption is neglected and a homogeneous plane wave source is assumed, a quantifier for the intensity inhomogeneity, $\mathcal{I}_{1}$, can be formulated as

$$
\mathcal{I}_{I}=\frac{1}{V I_{0}} \int\left|I_{0}-I(x)\right| d V
$$

where $V$ denotes the considered volume, $I_{0}$ and $I(x)$ are the (uniform and constant) source intensity and composite intensity field, respectively, and the brackets $|\cdot|$ indicate the absolute value of the term in between. Note that Eq. (14) equals the volume average of the absolute intensity difference compared to the unfilled resin.

The light intensity is related to the photopolymerization through the polymerization rate, i.e. the conversion rate of monomer [12]:

$$
R_{p}=-\frac{d M}{d t}=\mathcal{P}_{M} \sqrt{I} M
$$

where $M$ is the monomer concentration and $\mathcal{P}_{M}$ is a lumped polymerization factor. $\mathcal{P}_{M}$ can be a function of conversion, initiator, inhibitor, temperature, etc. [37-40]. The degree of conversion is defined as the concentration of monomer that has been converted into polymer normalized to the initial monomer concentration, i.e. [38]

$$
p=\frac{M_{0}-M}{M_{0}},
$$

with $M_{0}$ the initial monomer concentration. To quantify the polymerization due to the inhomogeneous intensity field (compared to the homogeneous unfilled situation), a second inhomogeneity quantification term is formulated and termed the conversion based intensity effectiveness:

$$
\mathcal{E}_{p}=\frac{1}{V} \int \frac{e-\exp \left(1-\sqrt{(x) / I_{0}}\right)}{e-1} d V
$$

where $e=\exp (1)$. Note that $\exp \left(-\sqrt{I(x) / I_{0}}\right)$ in Eq. (17) is the functional term motivated by Eqs. (15) and (16), whereas the remaining terms are merely introduced to ensure $\mathcal{E}_{p}\left(I(x)=I_{0}\right)=1$ and $\mathcal{E}_{p}(I(x)=0)=0$.

\section{Analysis of the role of the inclusion shape}

The influence of the inclusion shape is first analyzed in a two-dimensional framework. The motivation for this lies in the numerical efficiency of the analysis compared to a three-dimensional formulation. The question whether the resulting conclusions translate to 3D is addressed in Section 4. The analysis is first conducted on an isolated particle, subsequently moving to a more realistically filled resin.

\subsection{Model formulation}

The mathematical framework described in the previous section allows for an electromagnetic analysis of a particle-filled resin. Focus is put on isolating the effect of the inclusion shape, initially through a single-scattering analysis.

\subsubsection{Isolated shape analysis}

To parametrize and systematize the two-dimensional shape analysis, regular convex polygons of order $n$ are used, as displayed in Fig. 3. The volume of the different shapes is equal to that of the $1 \mu \mathrm{m}$ diameter circular shape denoted with $n=\infty$.

To accommodate the scatter-analysis, the electric field $\vec{E}$ is split into a background plane-wave excitation field $\vec{E}_{0}$ and a resulting scattered field $\vec{E}_{s}$, i.e. $\vec{E}=\vec{E}_{0}+\vec{E}_{s}$, while only solving the latter [41]. To do so, the scatterer (which is embedded in 

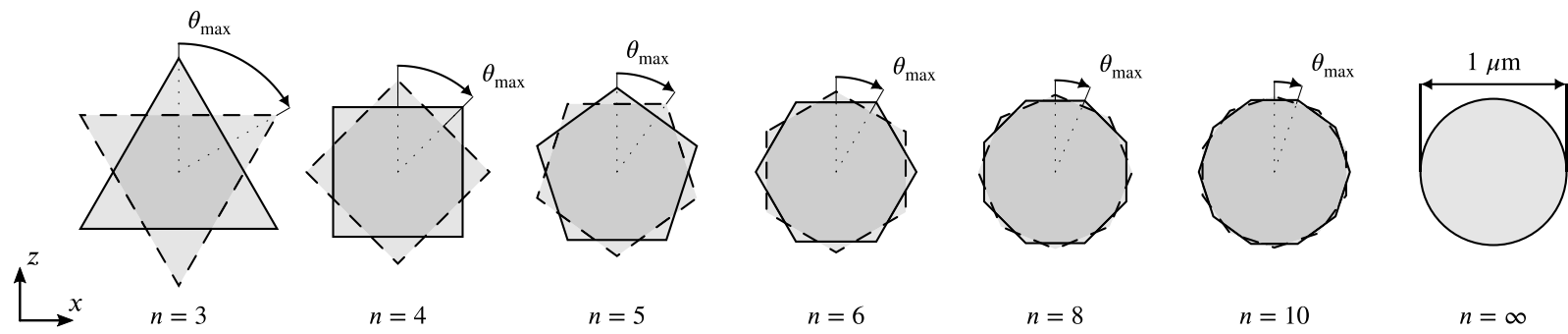

Fig. 3. Different polygonal shapes with identical volume of which the orientation is specified by the unique rotation angle $\theta_{\text {max }}$ (denoted by the arrow).

Table 1

Considered material properties and model parameters.

\begin{tabular}{llll}
\hline Parameter & Value & Unit & Description \\
\hline$r_{\circ}$ & 0.5 & {$[\mu \mathrm{m}]$} & Radius of spherical particle \\
$n_{r}$ & 1.5 & {$[-]$} & Refractive index of resin \\
$n_{c}$ & 1.78 & {$[-]$} & Refractive index of alumina powder \\
$\lambda$ & 400 & {$[\mathrm{~nm}]$} & Wavelength of UV light in vacuum \\
$I_{0}$ & 100 & {$\left[\mathrm{~W} / \mathrm{m}^{2}\right]$} & Light source intensity \\
\hline
\end{tabular}

the domain of interest) is bounded by an artificial layer, where the PML is applied [42]. Note that in contrast to the boundary conditions illustrated in Fig. 2, here a PML surrounds the whole physical domain along all four edges. The physical embedded domain has dimensions of $x \times z=8 \times 8 \mu \mathrm{m}^{2}$, with the inclusion centered in the horizontal middle and $2 \mu \mathrm{m}$ from the top. The light with intensity $I_{0}$ is propagating from a uniform source in the negative $z$-direction, i.e. from top to bottom. This configuration puts the scatterer close to the light source with sufficient space on the other side to display the resulting inhomogeneity. The width is also chosen $8 \mu \mathrm{m}$ to capture scattering effects in the direction perpendicular to the illumination resulting from different orientations. These domain dimensions were found to provide converged trends in the results, without being unnecessarily large. To investigate the effect of the inclusions' orientation with respect to the incoming light, they are rotated over an angle $\theta=0$ to $\theta=\theta_{\max }$ as illustrated in Fig. 3, where $\theta_{\max }=\pi / n$ rad. Note that this is only half of the polygon's rotation symmetry angle due to symmetry of the defined problem.

\subsubsection{Multiple-scattering}

Although the model with a single inclusion provides a clear insight into the effect of the orientation of an isolated particle, a typical slurry will have a high filling content of particles with an arbitrary orientation. To investigate the cumulative effect of many scattering events, a multi-particle model is also used. The physical domain in this model is $x \times z=6.5 \times 11.5 \mu \mathrm{m}^{2}$ and contains 38 inclusions, such that the filling fraction equals $\phi=0.40$. Uniformly distributed light enters the domain through the top edge with intensity $I_{0}$. The left and right boundaries are coupled through Bloch-Floquet periodic boundary conditions and the domain is extended through a PML at the bottom, see Fig. 2.

\subsubsection{Material properties and model parameters}

The required material properties for the models in Sections 3.1.1 and 3.1.2 are provided in Table 1 and representative for a typical acrylic resin with alumina powder as the ceramic filler. In the considered domains, the absorption of the resin has been neglected because its effect would be insignificant within the considered length scales [12]. At these wavelengths the absorption of the ceramic inclusions can also be neglected [5]. The applied light source intensity $I_{0}$ and wavelength $\lambda$ are also specified in Table 1 .

\subsection{Results}

Before presenting the more representative case of multiple-scattering in Section 3.2.2, the impact of a single inclusion's shape and orientation on the light propagation is addressed first.

\subsubsection{Analysis on isolated particle}

The different inclusions displayed in Fig. 3 are rotated over 13 unique angles from $\theta_{1}=0$ to $\theta_{13}=\theta_{\max }$. The resulting total intensity field in the $8 \times 8 \mu \mathrm{m}^{2}$ domains for the intermediate rotation angle $\left(\theta_{7}\right)$ is displayed in Fig. 4. The graphs illustrate the influence of the inclusions on the intensity field $I$, compared to the initially homogeneous intensity distribution $I_{0}$, which is used to normalize the results. Notice that already for polygons with $n=6$ and higher, there is no clear (visible) difference in the resulting field compared to the circular inclusion. In order to quantify the effect of the shape and orientation of the particles, Fig. 5 shows the resulting intensity inhomogeneity parameter $\mathcal{I}_{I}$. The graph reveals several interesting trends. First of all, the peak inhomogeneity for all shapes coincides with the maximum rotation angle. By the choice of 

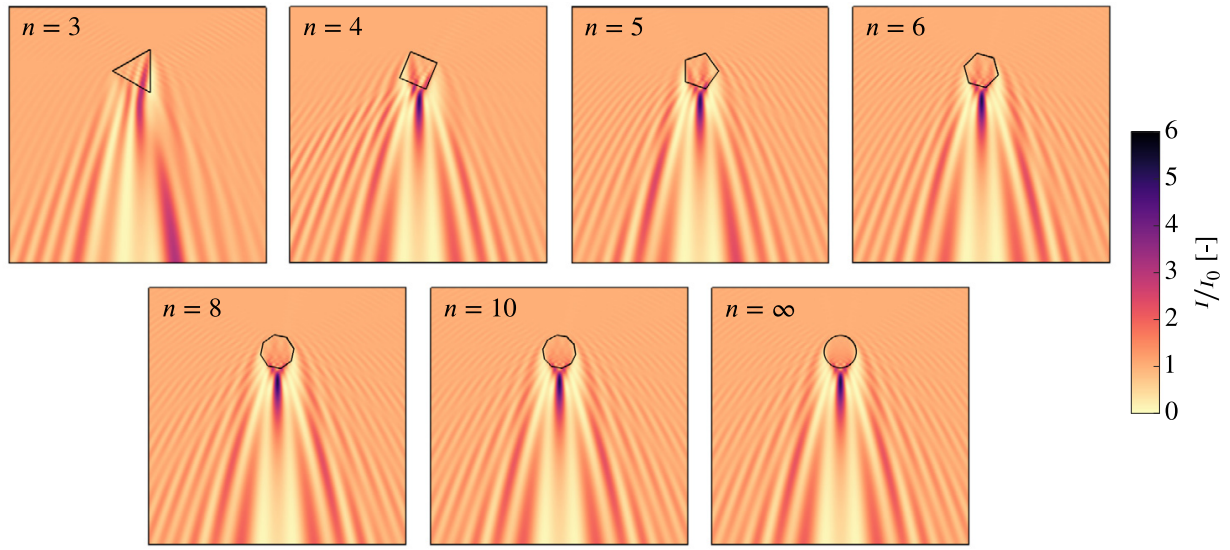

Fig. 4. Normalized intensity field $I / I_{0}$ in the $8 \times 8 \mu \mathrm{m}^{2}$ domain for $n=3,4,5,6,8,10$ and $\infty$ at $\theta_{7}=\pi / 2 n$.

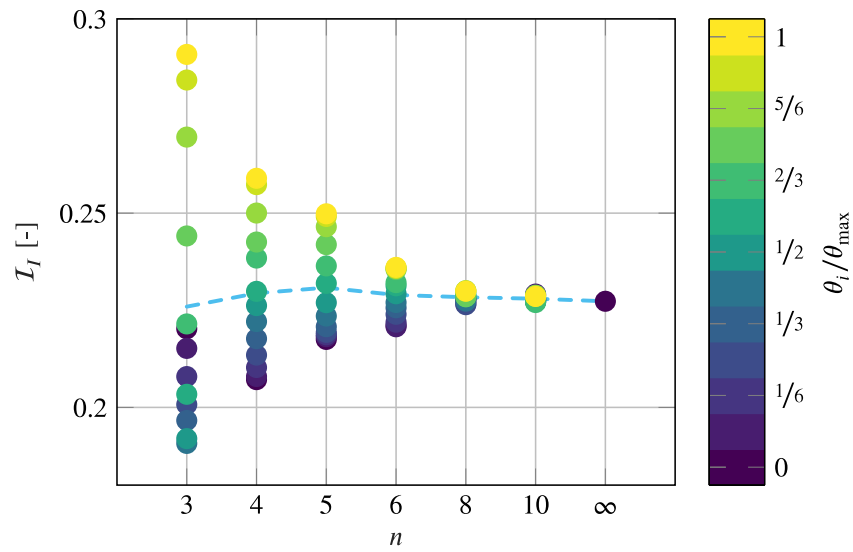

Fig. 5. $\mathcal{I}_{I}$ distribution for the different polygonal shapes over 13 equidistant angles from $\theta=0$ to $\theta_{\max }$. The average value is indicated by the blue dashed line.

the initial orientation, i.e. with an edge facing downwards (cf. Fig. 3), the peak intensity at $\theta_{\max }$ thus corresponds to a corner pointing in the direction of light propagation. Secondly, as illustrated by the average $\mathcal{I}_{I}$ (the dashed blue line in Fig. 5), the corresponding value largely coincides with the result for the intermediate rotation angle as depicted in Fig. 4. Additionally, the dominant trend in the angle dependency is a decrease in $\mathcal{I}_{I}$ for $\theta \rightarrow 0$. The exception here is the triangular particle, which for increasing rotation angles initially shows a decrease followed by a steeply increasing inhomogeneity. Overall, the bandwidth of $\mathcal{I}_{I}$-values increases with a reduction in the polygon-order $n$. The latter two trends are further highlighted in Fig. 6, which displays $\mathcal{I}_{I}$ as a function of the actual orientation angle. Whereas all lines for $n>3$ initially have a positive slope, the line with $n=3$ initially descends. Fig. 6 also reflects the rotational symmetry for the intensity inhomogeneity of the different polygons analyzed.

An alternative intensity inhomogeneity quantification parameter, linked to its effect on the conversion, was introduced in Section 2.4, termed $\mathcal{E}_{p}$, for which the results are provided in Fig. 7. Considering both Figs. 5 and 7, similar trends emerge with respect to, e.g., range of $\mathcal{I}_{I}$ and $\mathcal{E}_{p}$ values for the corresponding polygon order. Whereas $\mathcal{I}_{I}$ did not show a clear trend with respect to the average inhomogeneity for increasing polygon order, the average $\mathcal{E}_{p}$ does show a clear improvement of conversion effectiveness for increasing $n$. The orientation dependence for $\mathcal{E}_{p}$ appears to be of opposite sign compared to that of $\mathcal{I}_{I}$, indicating that while the inhomogeneity in the intensity-field decreases, the conversion effectiveness increases.

\subsubsection{Analysis on multiple-scattering}

The case of a single inclusion is not representative for a slurry with a high solid filling fraction. Here, a filling fraction of $\phi=0.40$ is considered for 50 different realizations of randomly positioned and oriented polygons of a single type in a horizontally periodic $6.5 \times 11.5 \mu \mathrm{m}^{2}$ domain. Three realizations for the different polygons $(n)$ are depicted in Fig. 8, including the resulting intensity field. At first glance, no characteristic features can be identified that are coherent for a specific polygonal order. To scrutinize possible hidden trends in these results, a statistical analysis is performed on the 

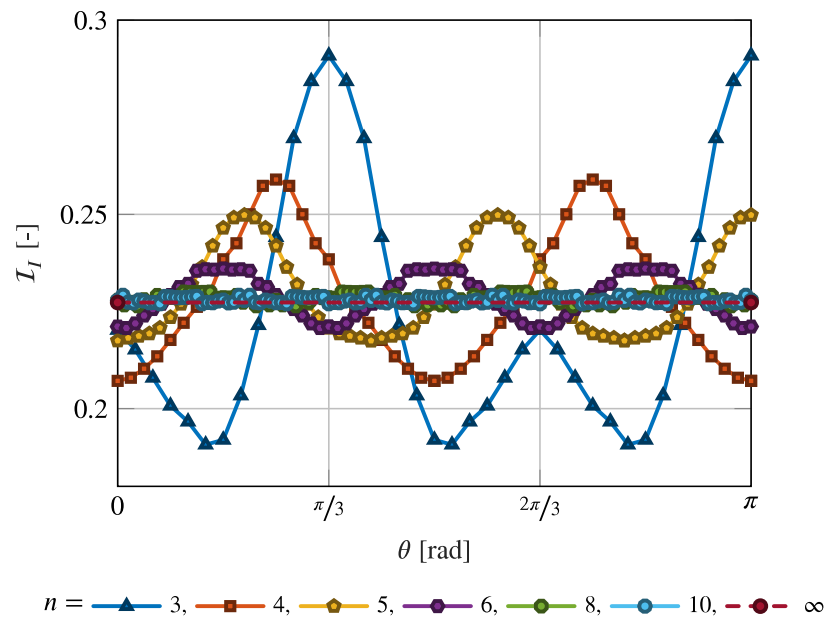

Fig. 6. $\mathcal{I}_{I}$ as a function of the inclusion orientation $\theta$.

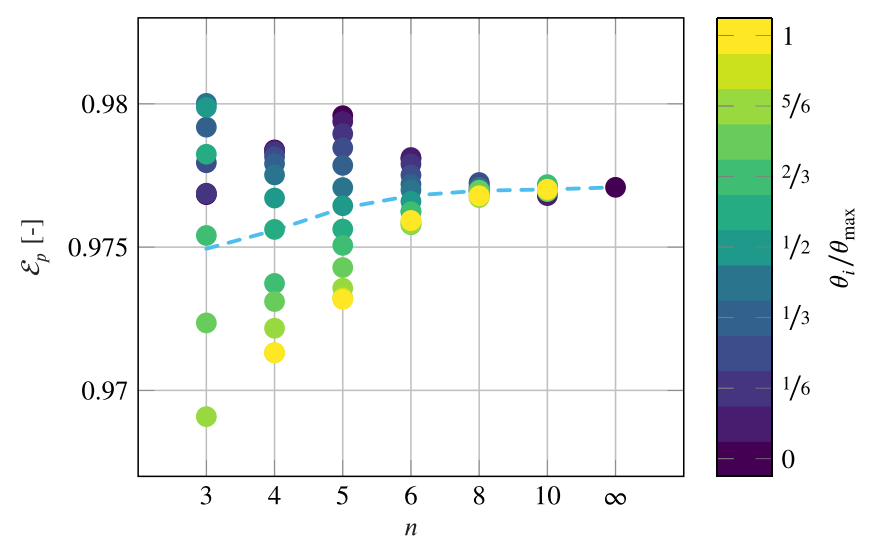

Fig. 7. $\mathcal{E}_{p}$ distribution for the different polygonal shapes over 13 equidistant angles along $\theta_{\max }$. The average value is indicated by the blue dashed line.

inhomogeneity parameters. The results of this analysis are displayed in Fig. 9. Note that the horizontal spread for each $n$ has only been added to reveal overlapping values. For each polygon shape the central red line inside the boxes indicates the mean value and the light red and blue box represent the standard error of the mean within a 95\% confidence interval and the standard deviation, respectively. Based on Fig. 9, no trends can be identified in either $\mathcal{I}_{I}$ or $\mathcal{E}_{p}$. It has been verified that there is no inclination towards certain orientation angles for any of the polygons, i.e. the inclusions are randomly oriented. Note that the multiple-scattering significantly increases the inhomogeneity in the intensity $\mathcal{I}_{I}$ compared to the single scattering situation, but this trend is not repeated by $\mathcal{E}_{p}$, implying that although the inhomogeneity in the intensity field increases, the average conversion effectiveness is analogous to the single-scattering case. An interesting finding is the occurrence of $\mathcal{E}_{p}$-values larger than 1 , which are attributed to the choice of model (both material and boundary) conditions. Due to the introduction of multiple-scattering and the periodic boundary conditions, the incoming light is partially refracted towards the horizontal direction, causing the average intensity in the domain to increase. The resulting higher peak intensity (cf. Fig. 8) in turn causes the increased $\mathcal{I}_{1}$-values. Note that the average in- and out-flow components of the intensity $\left\langle S_{y}\right\rangle$ are still equivalent to $I_{0}$. Considering a non-periodic setting this convincingly illustrates the effect of broadening of the cure profile as observed experimentally. Even though the scattering events (in these infinitely periodic domains) increase the local average intensity, the mean $\mathcal{E}_{p}$-value is still below 1 , i.e. a homogeneous intensity profile equal to $I_{0}$ still leads to more effective photopolymerization. Note that the corresponding homogeneous conversion would also be preferential considering the absence of residual monomer.

\section{Three-dimensional analysis}

Multiple inclusion simulations similar to the ones illustrated in Fig. 8 quickly become computationally very demanding in a three-dimensional framework. To alleviate this, it will therefore be verified whether the dominant trends in twodimensions (qualitatively or quantitatively) can be extrapolated to three-dimensional powder processes. 

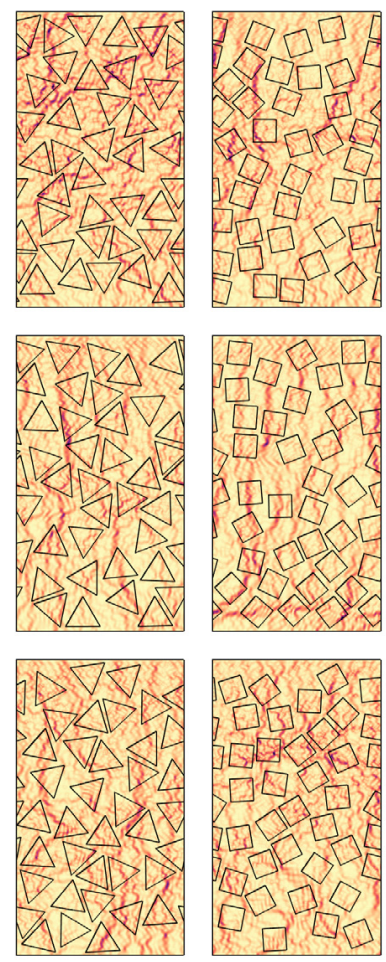
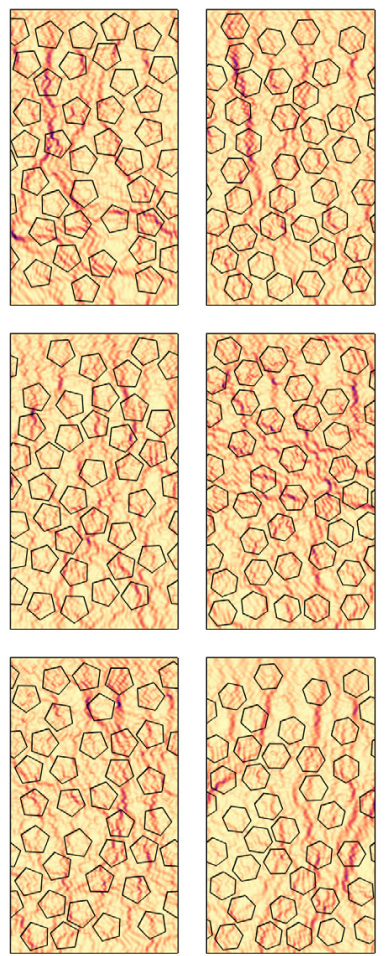
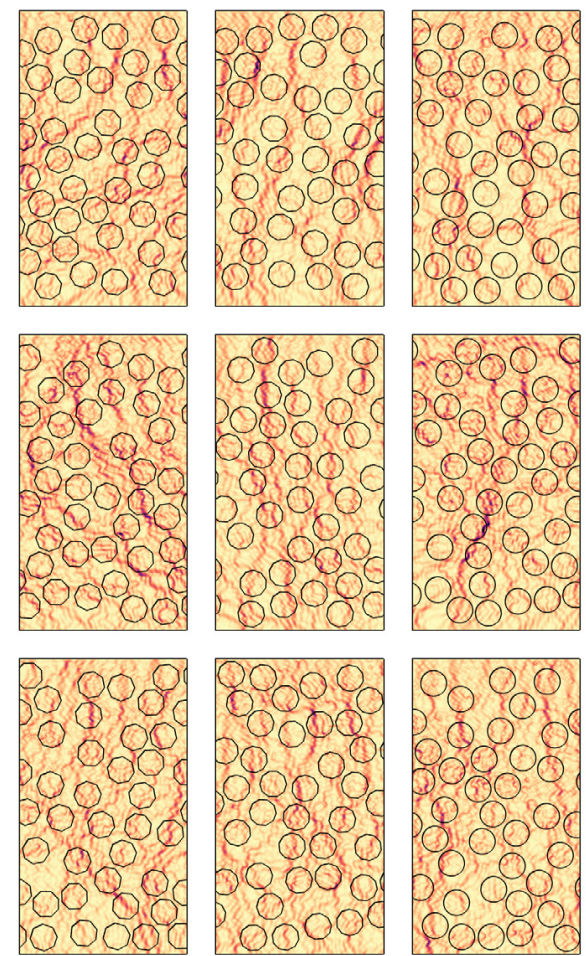

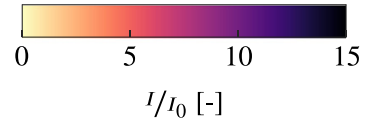

Fig. 8. Intensity field for (from left to right) $n=3,4,5,6,8,10$ and $\infty$ for three different random realizations.
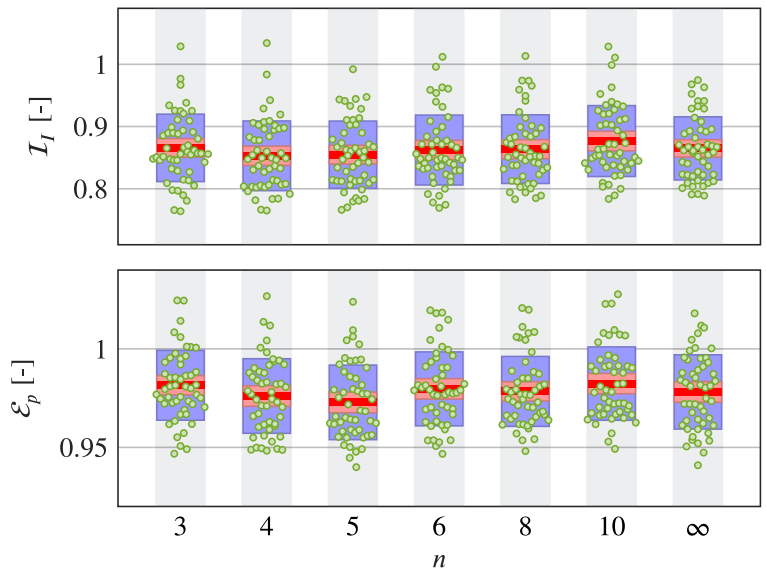

Fig. 9. Distribution of $\mathcal{I}_{I}$ and $\mathcal{E}_{p}$ for 50 random realizations per polygonal shape. For each shape, the result of the 50 realizations is displayed by the markers. The red line indicates the average value, the light red box its $95 \%$ confidence interval and the blue box the standard deviation. (For interpretation of the references to color in this figure legend, the reader is referred to the web version of this article.)

\subsection{Model formulation}

The most logical extension translating the two-dimensional regular, convex polygons to three-dimensional particles is to use platonic solids, which are regular, convex polyhedra. In total there are five of those geometries, i.e. the tetrahedron, hexahedron or cube, octahedron, dodecahedron and icosahedron [45]. Here, the latter four, as illustrated in Fig. 10, are considered and compared to the perfect sphere. The sizes are scaled such that the volumes of all considered geometries 


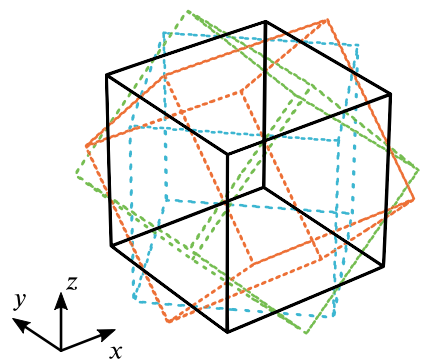

(a) Hexahedron $(12,6)$

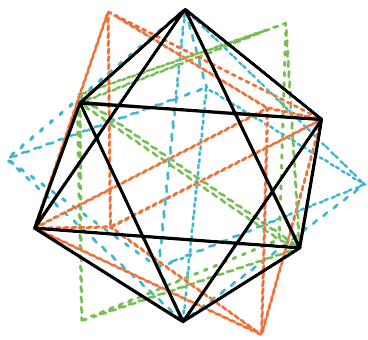

(b) Octahedron $(12,8)$

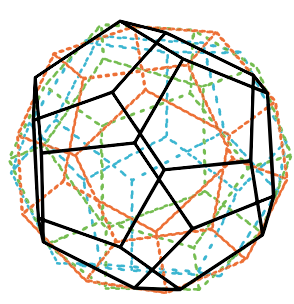

(c) Dodecahedron $(30,12)$

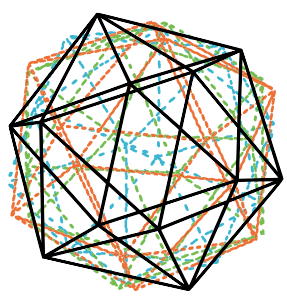

(d) Icosahedron $(30,20)$

Fig. 10. Platonic solids and the considered orientations (along the major axis) with the number of edges $e$ and faces $f$ denoted as ( $e, f$ ).

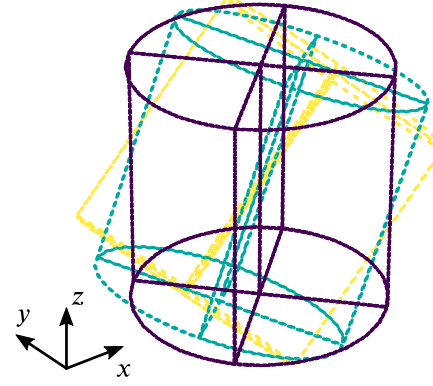

(a) $n=4, \theta_{0}=0$

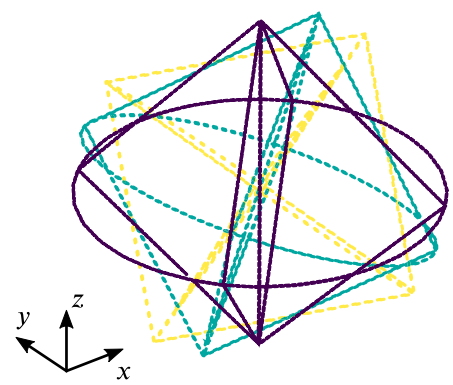

(e) $n=4, \theta_{0}=\theta_{\max }$

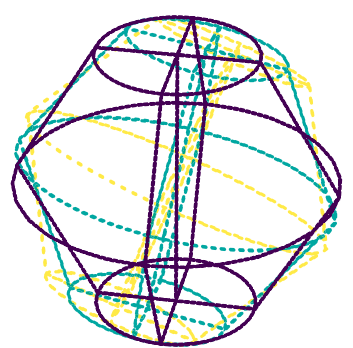

(b) $n=6, \theta_{0}=0$

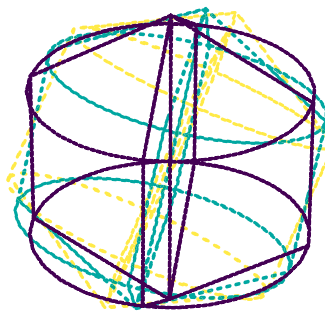

(f) $n=6, \theta_{0}=\theta_{\max }$

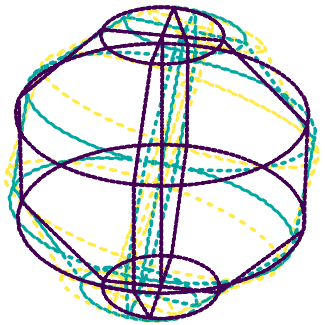

(c) $n=6, \theta_{0}=0$

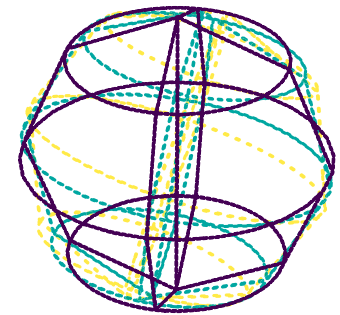

(g) $n=8, \theta_{0}=\theta_{\max }$

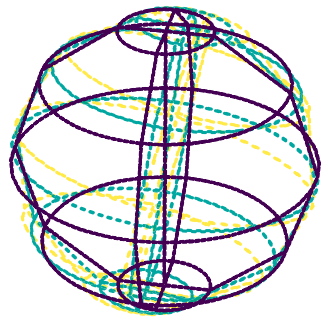

(d) $n=10, \theta_{0}=0$

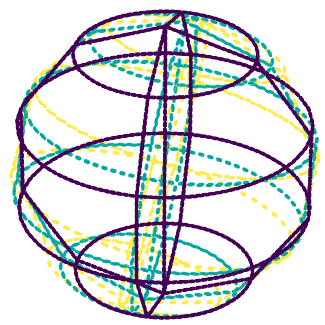

(h) $n=10, \theta_{0}=\theta_{\max }$

Fig. 11. Two-dimensional shapes revolved around the $z$-axis in a 3D shape, by using the 2D configuration with either $\theta_{0}=0$ (Figs. a-d) or $\theta_{0}=\theta_{\text {max }}$ (Figs. e-h). Shape color corresponds to the $\theta_{i} / \theta_{\max }$ bar in Figs. 5, 7 and 13.

are equal to that of the sphere with $0.5 \mu \mathrm{m}$ radius. Whereas the hexahedron and octahedron and the dodecahedron and icosahedron have the same number of edges, respectively 6 and 12, the number of faces increases from $6,8,12$ to 20 . Consequently, judging from the number of faces, from left to right in Fig. 10 the shapes are ordered to more and more resemble the sphere. Whereas in a two-dimensional simulation the orientation of the inclusions can only be varied along the out-of-plane $y$-axis, now two perpendicular axes, $x$ and $z$, can also be considered. The initial inclusion orientation (the black solid line in Fig. 10) with its local 1-, 2- and 3-axis aligned with the global $x$-, $y$ - and $z$-axis, respectively, is rotated around the $x-, y$ - and $z$-axis. The rotations over the major axes are illustrated by the dashed lines in Fig. 10.

In order to provide a direct relationship between the two-dimensional results and the corresponding three-dimensional representation, a second series of 3D shapes is considered. In converting the 2D geometries from Fig. 3 to 3D, instead of performing an out-of-plane extrusion, the even- $n$ shapes are revolved around the $z$-axis. This revolution is both applied to the initial geometry with $\theta_{0}=0$ and the geometry with $\theta_{0}=\theta_{\max }$ (angles in Fig. 3). The resulting three-dimensional geometries are shown for $\theta_{0}=0$ and $\theta_{0}=\theta_{\max }$ in Figs. 11a to 11d and Figs. 11e to 11h, respectively. The three-dimensional shapes will next be rotated around the $y$-axis (equivalent to the two-dimensional analysis) over angles from $\theta=0$ to $\theta=\theta_{\max }$ as indicated in Fig. 11 by the lighter wireframe geometries at $\theta=\frac{1}{2} \theta_{\max }$ and $\theta_{\max }$. 

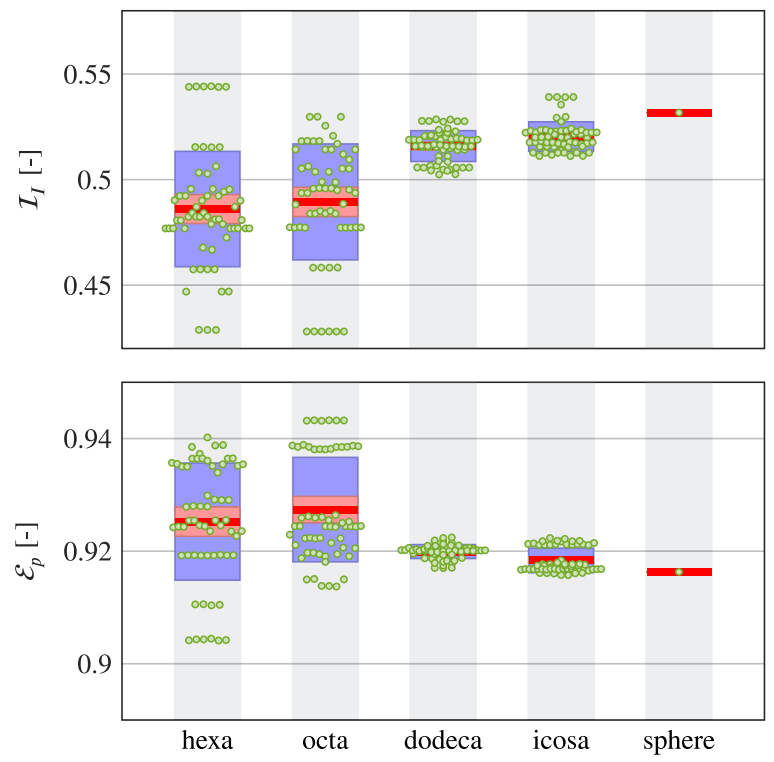

Fig. 12. $\mathcal{I}_{l}$ and $\mathcal{E}_{p}$ results for the three-dimensional shapes for rotations over the $x-, y$ - and $z$-axis of angles in range from $[0: 30 / 45: 90]$ deg. The red line indicates the average value, the light red box its $95 \%$ confidence interval and the blue box the standard deviation. (For interpretation of the references to color in this figure legend, the reader is referred to the web version of this article.)

For both three-dimensional models, i.e. with platonic solid inclusions and revolved geometries, the computational domain is limited to $x \times y \times z=1.5 \times 1.5 \times 2.5 \mu \mathrm{m}^{3}$, with periodic boundary conditions on the $x z$ - and $y z$-planes. The light enters the domain from the top and the bottom surface is extended by an absorbing PML, in accordance with Fig. 2. The inclusion is placed $0.75 \mu \mathrm{m}$ from the top surface in the horizontal middle and model parameters follow from Table 1 .

\subsection{Results}

The platonic shapes as described in Section 4.1 are analyzed first to examine the influence of geometry and orientation. A comparison to two-dimensional results follows in Section 4.2.2.

\subsubsection{Platonic solid geometries}

Compared to the initial orientation, the black solid line in Fig. 10, the platonic shapes are rotated around the $x, y$ and $z$-axis over intervals from 0 to 90 degrees. Consequently, at least a full rotational symmetry is captured for the individual shapes. The $0-90$ degree interval is subdivided into both $[0,30,60,90]$ and $[0,45,90]$ degree combinations. This does not provide a full orientation map, but still involves $4^{3}+3^{3}=91$ possible orientations (not subtracting multiply defined combinations). To further reduce this orientation set, the considered orientations are limited to the ones with a uniquely oriented 3-axis. This limiting choice implies that for each 3-axis orientation, only a single 1-and 2-axis orientation is evaluated. Here, the convention is used that the local inclusion axes initially correspond to the global axes, i.e without rotation the local 1,2 and 3-axis coincide with the $x, y$ and $z$-axis, respectively. A total number of 61 orientations, i.e. simulations, were finally considered to investigate the shape orientation dependence of the platonic solids. It is interesting to emphasize the resulting computational demand for these. Whereas the two-dimensional simulations can still be performed on a portable system with $8 \mathrm{~GB}$ of RAM using a direct solver for $\mathcal{O}(2.5$ million) degrees-of-freedom, the three-dimensional simulations considered here require approximately $60 \mathrm{~GB}$ of RAM using a GMRES iterative solver with a multi-grid preconditioner for $\mathcal{O}(8$ million) degrees-of-freedom.

The results of this analysis are visualized in Fig. 12 for both $\mathcal{I}_{I}$ and $\mathcal{E}_{p}$. The result for the sphere is also included for comparison. Note that the restricted range of angles may not provide a full statistical analysis, but it is merely used to identify the spread in the obtained results. Similar to previous graphs, the central red line indicates the mean value and the light red and the blue box represent the standard error of the 95\% confidence interval of the mean and the standard deviation, respectively. The horizontal spacing between data points only serves to separate overlapping data points. Note that a quantitative comparison to the results of the two-dimensional isolated shape analysis should not be done here, given the reduced domain size and periodic boundary conditions applied here. The physical geometry reflects a horizontal layer with inclusions arranged in a regular grid. Consequently, neighboring particles do influence the light propagation and, although periodic at the particle scale, more resembles the multiple-scattering case.

Fig. 12 shows a trend of increasing inhomogeneity $\mathcal{I}_{I}$ from left to right, so the order hexahedron-octahedrondodecahedron-icosahedron indeed converges to the sphere. Equivalently, the spread in results narrows towards the sphere, 
which is independent of the orientation. The distinction between the number of edges, 12 versus 30 for the former and latter two shapes, can also be identified from the spread in data-points. Comparing the hexa with the octa and the dodeca with the icosa, both pairs have a similar bandwidth with respect to the average confidence interval and the standard deviation. Similar conclusions can also be drawn from the $\mathcal{E}_{p}$-values, although the reduction in $\mathcal{I}_{I}$ translates to an increasing effectiveness $\mathcal{E}_{p}$. For these boundary conditions there appears to be a preference for lower order polyhedra, which contradicts the isolated two-dimensional analysis in Fig. 7. In comparison to the two-dimensional multiple-scattering simulation (cf. Fig. 9), the smaller magnitude of $\mathcal{I}_{l}$-values indicates that the regular grid does not promote significant horizontal light propagation. As a consequence the conversion effectiveness is now further reduced compared to the homogeneous case. The set of orientation angles considered induces a clear separation between two clusters of $\mathcal{E}_{p}$-values for the icosahedron while for the hexahedron and octahedron multiple orientations exist with clustered or equal $\mathcal{E}_{p}$-values. For a complete angle dependency the reader is referred to the Appendix where Figs. A.14 and A.15 display the results for $\mathcal{I}_{I}$ and $\mathcal{E}_{p}$ in polar graphs. The results from the analysis on platonic solids provide interesting insights, but it remains interesting to deduce whether a two-dimensional analysis is adequate in representing a three-dimensional physical process.

\subsubsection{Revolved three-dimensional geometries}

To facilitate a 2D-3D comparison, the second series of geometries is considered next, see Fig. 11. In order to make the comparison to the 2D results, the results for the revolved shapes in Fig. 11 are compared to two-dimensional simulations on a $x \times z=1.5 \times 2.5 \mu \mathrm{m}^{2}$ domain which is also periodic in the horizontal direction. As a consequence, the two-dimensional crosssection in the $y=0$ plane is equal for the two- and three-dimensional analysis. Comparing the two- and three-dimensional results in fact implies a comparison between an extruded and a revolved three-dimensional polygon, respectively. The resulting $\mathcal{I}_{I}$ and $\mathcal{E}_{p}$-values are displayed in Fig. 13. The open circles denote the result from the two-dimensional model with periodic boundary conditions, which are clearly different from the results in Figs. 5 and 7 . A first prominent difference is the magnitude of the $\mathcal{I}_{I}$ and $\mathcal{E}_{p}$-values. The $\mathcal{I}_{I}$-values are significantly higher in this case, because the domain of interest is smaller and inclusions are more closely packed together. Such an increase of $\mathcal{I}_{I}$ was already identified in the multiplescattering simulations but also hold in the regular grid considered here. The $\mathcal{E}_{p}$-values on the other hand differ more from 1, i.e. the conversion based intensity effectiveness reduces. These findings indicate that although higher peak intensities exist, the average effective conversion decreases compared to the homogeneous domain or even the isolated particle case. The single exception to these conclusions is the point where the square $(n=4)$ is oriented at $\theta_{\max }$. In this orientation the corners of the square are close together, which results in a strong interaction between neighboring particles as reflected by both the $\mathcal{I}_{I}$ and $\mathcal{E}_{p}$-value. Similarly to some of the realizations in the two-dimensional multiple-scattering simulations, the effectiveness appears to be higher than in the homogeneous situation. This can again be attributed to the horizontal interactions and illustrates the significant effect of orientation for non-spherical particles. Another remarkable observation is that due to the difference in boundary conditions the trend in average $\mathcal{I}_{I}$ - and $\mathcal{E}_{p}$-values is opposite to the one in Figs. 5 and 7 , respectively. The slope of the $\mathcal{I}_{I}$ average shows opposite behavior compared to Fig. 5 and the effectiveness $\mathcal{E}_{p}$ improves for increasing polygon order. This clearly illustrates that using single scattering theories for multiple-scattering physics is not appropriate.

The high $\mathcal{I}_{1}$ - and $\mathcal{E}_{p}$-values for $n=4$ are not found in the three-dimensional simulations represented by the square and rhombus symbols in Fig. 13. This can most likely be attributed to the increase in average distance between the faces of neighboring particles. The squares in Fig. 13 correspond to revolved shapes with a side initially perpendicular to the incoming light (Figs. 11a to 11d), similar to the two-dimensional analysis, and the rhombus depicts results for revolved shapes with their corners initially aligned with the light direction (Figs. 11e to $11 \mathrm{~h}$ ). In a general sense, $\mathcal{I}_{\mathrm{I}}$-values are higher in a twodimensional setting but the bandwidth of results overlaps to a large extent with respect to $\mathcal{E}_{p}$. This indicates that although the inhomogeneity in the intensity field is overestimated in a two-dimensional setting, the effectiveness of the resulting conversion is equivalent. As expected from the initial orientation similarity, the orientation angle dependence of the shapes with $\theta_{0}=0$ is fairly similar to the two-dimensional analysis. This is more obvious for $\mathcal{E}_{p}$ than for $\mathcal{I}_{l}$, where $n=4$ and 6 do not show this dependence. The $\mathcal{I}_{1}$ - and $\mathcal{E}_{p}$-values for the revolved shapes with $\theta_{0}=0$ and $\theta_{0}=\theta_{\max }$ are highly analogous. Compared to the platonic shapes, the $\mathcal{I}_{I}$-values are slightly higher (on average) for the revolved shapes, but the $\mathcal{E}_{p}$ is of the same order suggesting equivalent conversion effectiveness for all considered three-dimensional shapes. A conclusion that can be drawn from Fig. 13 is that although the intensity profile might be different on the local scale, the effective emanating conversion in a three-dimensional setting is approximated correctly by a two-dimensional analysis.

\section{Conclusions}

The role of the powder in the additive manufacturing process for ceramics cannot be neglected. Clear effects were already identified in the initial attempts to print ceramics through vat photopolymerization, e.g. the pronounced broadening of the cure profile [5]. This work aimed to identify and quantify the effects introduced by particles in the VP process for ceramics, using a physics-based numerical modeling approach.

The numerical framework solves Maxwell's electromagnetic equations to capture the relevant effects emerging when the size of the particles is of the same order as the wavelength. From the resulting electric and magnetic vector field quantities, the field variable of interest can be obtained, i.e. the intensity or irradiance, which is the driving force for the photopolymerization reaction. The difference in refractive index combined with the inclusion geometry induces a large 


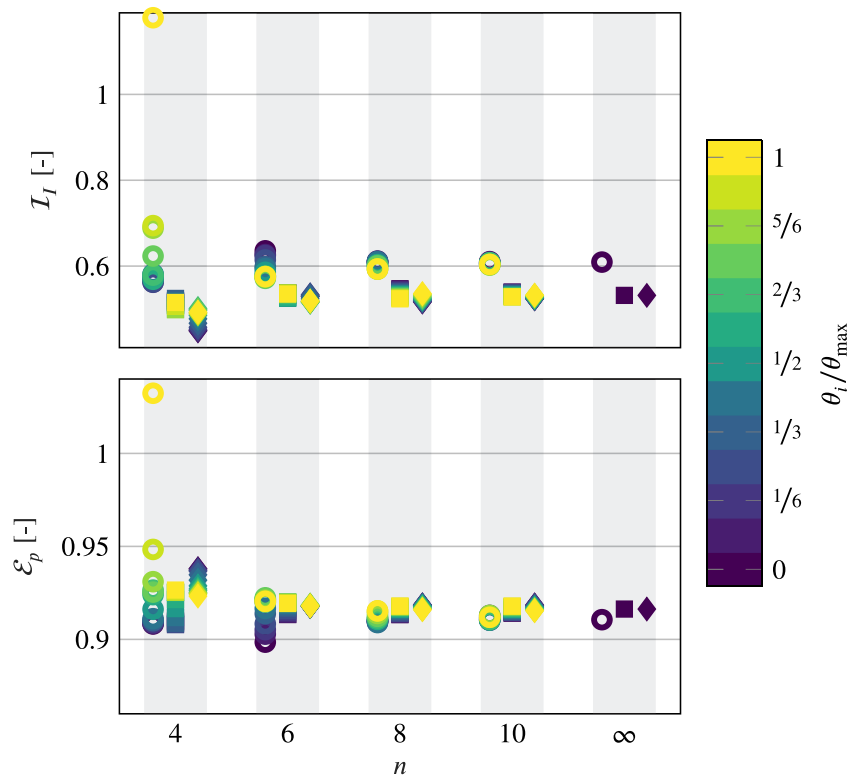

Fig. 13. Results of the two-dimensional versus three-dimensional revolved shape analysis. Circles are the 2D results, the squares correspond to 3D shapes with a side initially perpendicular to the incoming light (Figs. 11a to 11d) and the rhombus depicts 3D shapes with corners initially aligned with the light direction (Figs. 11e to 11h).

inhomogeneity in the intensity field due to the combined effects of reflection, refraction, interference and diffraction. The latter two physical effects can only be captured through a full wave description of the light. In order to quantify the effect of the inclusions, two unique intensity-based quantities are defined. The first one, the intensity inhomogeneity $\mathcal{I}_{1}$, quantifies the deviation from the disturbed intensity field compared to the uniform homogeneous case. The second one, the conversion based intensity effectiveness $\mathcal{E}_{p}$, relates the non-homogeneous intensity field to the emanating polymerization reaction, without introducing additional material parameters. To parametrize the powder shape, particles are represented by polygonal (in 2D) or polyhedral (in 3D) shapes. This illustrates the academic nature of the study, considering the possible degree of randomness in actual powder shape, but at the same time allows for a clear and insightful study of the emerging effects.

Models with a single two-dimensional inclusion in a large uniform domain showed a small but clear trend favoring a higher-order polygon with respect to $\mathcal{E}_{p}$, on the other hand $\mathcal{I}_{I}$ does not show a clear trend. With respect to orientation dependence, lower $\mathcal{I}_{I}$ but higher $\mathcal{E}_{p}$ are found when the orientation is such that a flat side is opposite and perpendicular to the light origin, as is the case for $\theta=0$ in the considered models. Moving towards the more realistic multiple-scattering situation, all influences of particle geometry vanishes and there is no statistical preference for one polygon shape over the other. A practical transformation of multiple particle models to a three-dimensional setting is computationally demanding. To restrict computational requirements, models with a single incorporated inclusion and prescribed periodicity in the direction perpendicular to the illumination direction are proposed. The difference of these horizontally stacked particles compared to the isolated case is already significant in a two-dimensional setting. No clear trend can be identified for $\mathcal{I}_{1}$, but the effectiveness $\mathcal{E}_{p}$ now increases for reducing polygon order. This finding illustrates the necessity of accounting for multiple-scattering. The considered three-dimensional shapes (both platonic and revolved polygons) show the same trend in $\mathcal{E}_{p}$, i.e. a reducing value for more sphere-like geometries. The intensity inhomogeneity $\mathcal{I}_{I}$ on the other hand increases towards the sphere. By comparing results of a two-dimensional simulation to three-dimensional equivalent revolved shapes, it becomes clear that the two-dimensional simulations do have a clear predictive value. Although the inhomogeneity in the intensity field is slightly overestimated in the two-dimensional framework, the correspondence is excellent compared to $\mathcal{I}_{I}=0$ for the homogeneous case. Furthermore, the photopolymerization effectiveness $\mathcal{E}_{p}$ is very analogous for both twoand three-dimensions. Considering the interest lies in the conversion homogeneity resulting from the illumination step, the trends in the $\mathcal{E}_{p}$-value are deemed more important.

This study provides interesting insights for light scattering in a ceramic-resin system where typical properties of alumina and photopolymer resin are used. Nonetheless, it allows for other particle-matrix systems as well. The study illustrates the relevance of a two-dimensional analysis, preventing the need for highly computationally demanding three-dimensional simulations. However, a more generalized three-dimensional domain, with randomized placement and orientation, still needs to be explored. 


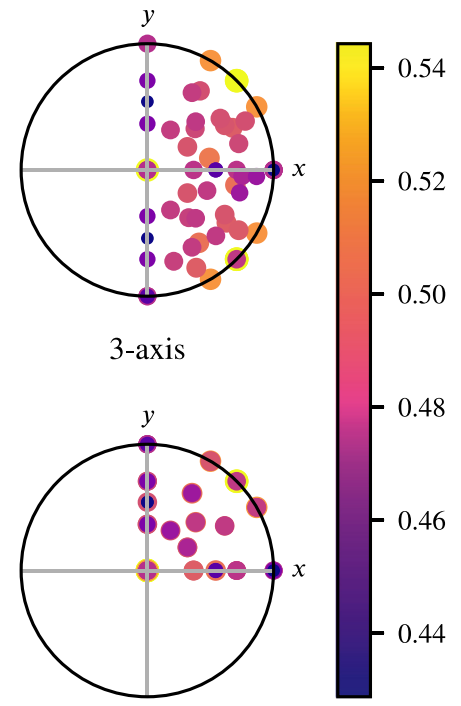

1 -axis

(a) Hexahedron (Fig. 10a)

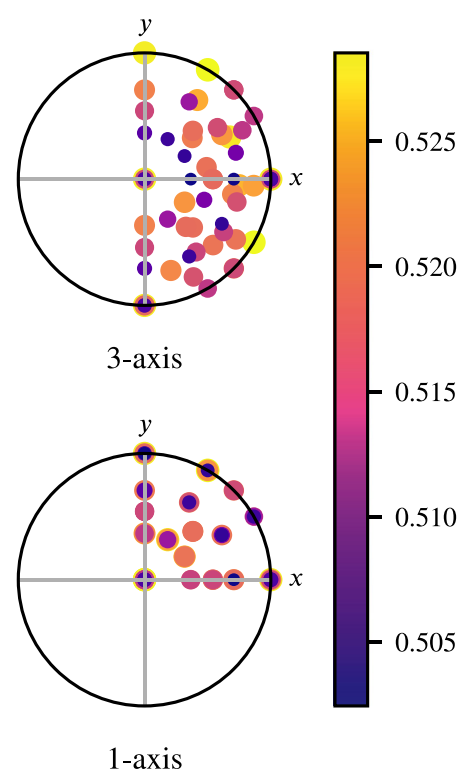

(c) Dodecahedron (Fig. 10c)

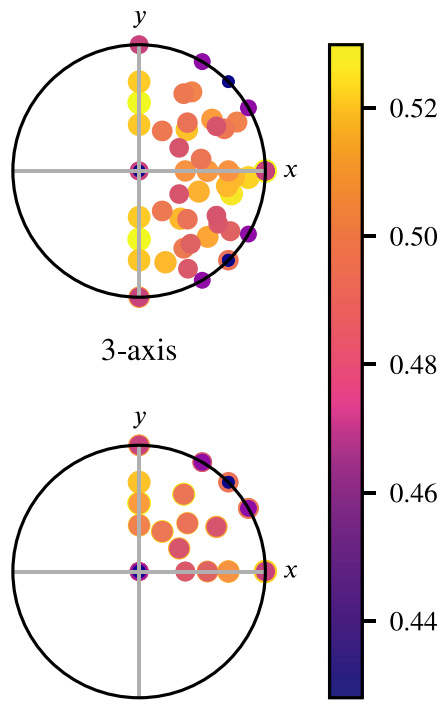

1-axis

(b) Octahedron (Fig. 10b)

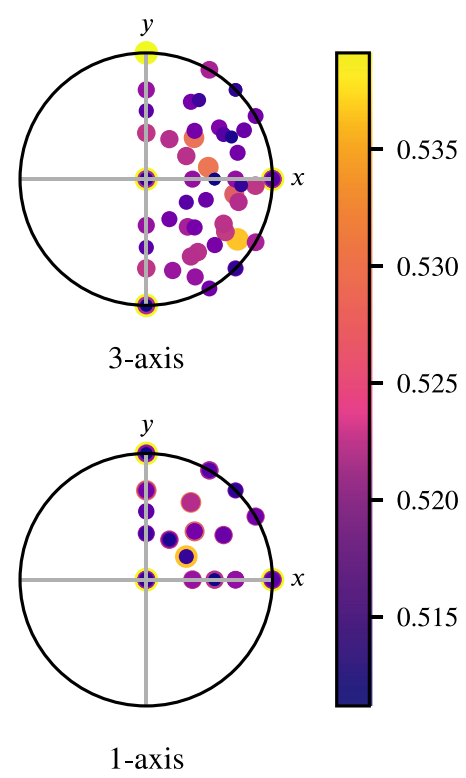

(d) Icosahedron (Fig. 10d)

Fig. A.14. Orientation dependent $\mathcal{I}_{I}$ for the platonic shapes. The magnitude of $\mathcal{I}_{I}$ is displayed for the orientation of the 3-and 1-axis (i.e. the axes initially coinciding with the global $x$ - and $y$-axis) using an equal area projection on the $x y$-plane.

\section{Acknowledgments}

This study was funded by the Netherlands Organisation for Applied Scientific Research (TNO), Netherlands and was carried out within the AMSYSTEMS Center, Netherlands. 


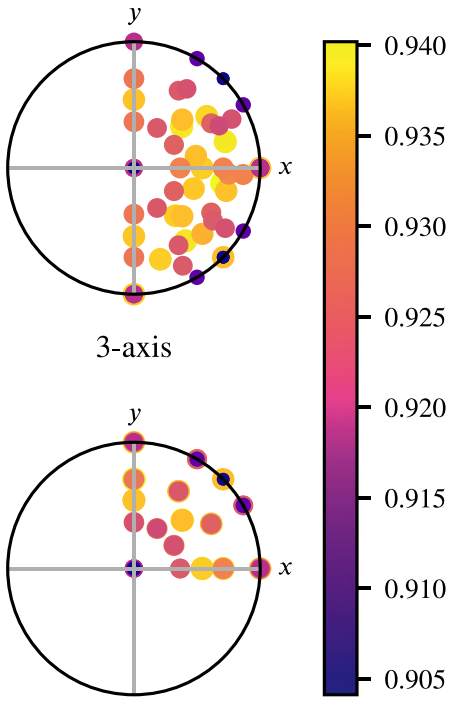

1 -axis

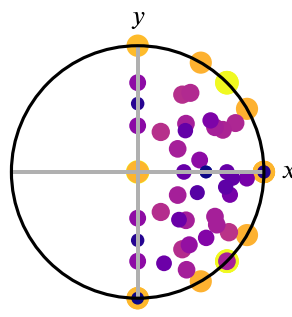

3-axis

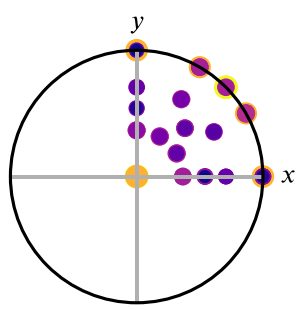

1 -axis

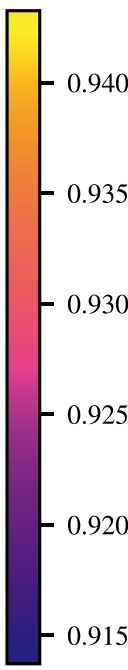

(b) Octahedron (Fig. 10b)

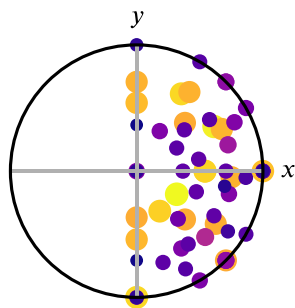

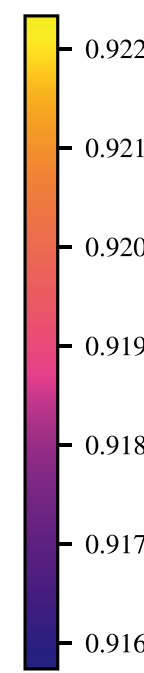

0.916 3-axis

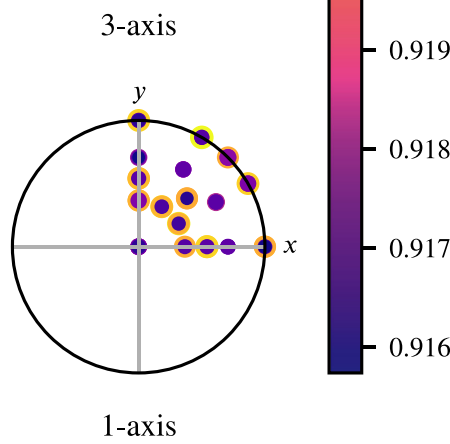

1 -axis

(d) Icosahedron (Fig. 10d)

1 -axis

(c) Dodecahedron (Fig. 10c)

Fig. A.15. Orientation dependent $\mathcal{E}_{p}$ for the platonic shapes. The magnitude of $\mathcal{E}_{p}$ is displayed for the orientation of the 3-and 1-axis (i.e. the axes initially coinciding with the global $x$-and $y$-axis) using an equal area projection on the $x y$-plane.

\section{Appendix. Orientation dependence in 3D}

The orientation dependent results corresponding to Fig. 12 are displayed in Figs. A.14 and A.15 for $\mathcal{I}_{I}$ and $\mathcal{E}_{p}$, respectively. The polar graphs show the orientation of the 3-and 1-axis, i.e. the local inclusion axis initially aligned with the $z$ - and $x$-axis, respectively. The marker sizes are scaled with the $\mathcal{I}_{I}$ and $\mathcal{E}_{p}$-value (corresponding to the marker color) such that the overlapping values are still uniquely visible. Note that the scales of the colorbar are not uniform to emphasize the orientation dependence. For the comparison between individual shapes, the reader is referred to Fig. 12. 


\section{References}

[1] A. Zocca, P. Colombo, C.M. Gomes, J. Günster, Additive manufacturing of ceramics: Issues, potentialities, and opportunities, J. Am. Ceram. Soc. 98 (7) (2015) 1983-2001, http://dx.doi.org/10.1111/jace.13700.

[2] J.R.G. Evans, Seventy ways to make ceramics, J. Eur. Ceram. Soc. 28 (7) (2008) 1421-1432, http://dx.doi.org/10.1016/j.jeurceramsoc.2007.12.015.

[3] J. Deckers, J. Vleugels, J.P. Kruth, Additive manufacturing of ceramics: A review, J. Ceram. Sci. Technol. 5 (4) (2014) 245-260, http://dx.doi.org/10.4416/ JCST2014-00032.

[4] P.F. Jacobs, Rapid Prototyping \& Manufacturing: Fundamentals of Stereolithography, Society of Manufacturing Engineers in cooperation with the Computer and Automated Systems Association of SME, Dearborn, MI, 1992, p. 434.

[5] J.W. Halloran, Ceramic stereolithography: Additive manufacturing for ceramics by photopolymerization, Annu. Rev. Mater. Res. 46 (2016) 10.1-10.22, http://dx.doi.org/10.1146/annurev-matsci-070115-031841.

[6] P. Colombo, G. Mera, R. Riedel, G.D. Sorarù, Polymer-derived ceramics: 40 Years of research and innovation in advanced ceramics, J. Am. Ceram. Soc. 93 (7) (2010) 1805-1837, http://dx.doi.org/10.1111/j.1551-2916.2010.03876.x.

[7] Y. Huang, M.C. Leu, J. Mazumder, A. Donmez, Additive manufacturing: Current state, future potential, gaps and needs, and recommendations, J. Manuf. Sci. Eng. 137 (2015) 014001, http://dx.doi.org/10.1115/1.4028725.

[8] V. Tomeckova, J.W. Halloran, Cure depth for photopolymerization of ceramic suspensions, J. Eur. Ceram. Soc. 30 (15) (2010) 3023-3033, http: //dx.doi.org/10.1016/j.jeurceramsoc.2010.06.004.

[9] S.P. Gentry, J.W. Halloran, Absorption effects in photopolymerized ceramic suspensions, J. Eur. Ceram. Soc. 33 (10) (2013) 1989-1994, http://dx.doi. org/10.1016/j.jeurceramsoc.2013.03.004.

[10] S.P. Gentry, J.W. Halloran, Depth and width of cured lines in photopolymerizable ceramic suspensions, J. Eur. Ceram. Soc. 33 (10) (2013) 1981-1988, http://dx.doi.org/10.1016/j.jeurceramsoc.2013.02.033.

[11] S.P. Gentry, J.W. Halloran, Light scattering in absorbing ceramic suspensions: Effect on the width and depth of photopolymerized features, J. Eur. Ceram. Soc. 35 (6) (2015) 1895-1904, http://dx.doi.org/10.1016/j.jeurceramsoc.2014.12.006.

[12] S. Westbeek, J.A.W. van Dommelen, J.J.C. Remmers, M.G.D. Geers, Multiphysical modeling of the photopolymerization process for additive manufacturing of ceramics, Eur. J. Mech. 71 (2018) 210-223, http://dx.doi.org/10.1016/j.euromechsol.2018.03.020.

[13] C.J. Bae, J.W. Halloran, Influence of residual monomer on cracking in ceramics fabricated by stereolithography, Int. J. Appl. Ceram. Technol. 8 (6) (2011) 1289-1295, http://dx.doi.org/10.1111/j.1744-7402.2010.02578.x.

[14] W. Zhou, D. Li, Z. Chen, The influence of ingredients of silica suspensions and laser exposure on UV curing behavior of aqueous ceramic suspensions in stereolithography, Int. J. Adv. Manuf. Technol. 52 (5-8) (2011) 575-582, http://dx.doi.org/10.1007/s00170-010-2746-8.

[15] K.C. Wu, J.W. Halloran, Photopolymerization monitoring of ceramic stereolithography resins by FTIR methods, J. Mater. Sci. 40 (1) (2005) 71-76, http://dx.doi.org/10.1007/s10853-005-5689-y.

[16] J.H. Nobbs, Kubelka-Munk theory and the prediction of reflectance, Rev. Prog. Color. Relat. Top. 15 (1) (1985) 66-75, http://dx.doi.org/10.1111/j.14784408.1985.tb03737.x.

[17] Y. Abouliatim, T. Chartier, P. Abelard, C. Chaput, C. Delage, Optical characterization of stereolithography alumina suspensions using the Kubelka-Munk model, J. Eur. Ceram. Soc. 29 (5) (2009) 919-924, http://dx.doi.org/10.1016/j.jeurceramsoc.2008.07.008.

[18] H. Liao, Stereolithography Using Compositions Containing Ceramic Powders (Ph.d. thesis), University of Toronto, 1997, p. 170.

[19] C. Sun, X. Zhang, The influences of the material properties on ceramic micro- stereolithography, Sensors Actuators 101 (2002) 364-370.

[20] C. Sun, X. Zhang, Experimental and numerical investigations on microstereolithography of ceramics, J. Appl. Phys. 92 (8) (2002) 4796-4802, http: //dx.doi.org/10.1063/1.1503410.

[21] E.S. Thiele, R.H. French, Light-scattering properties of representative, morphological rutile titania particles studied using a finite-element method, J. Am. Ceram. Soc. 81 (3) (1998) 469-479, http://dx.doi.org/10.1111/j.1151-2916.1998.tb02364.x.

[22] C. Leiner, W. Nemitz, S. Schweitzer, F.P. Wenzl, P. Hartmann, U. Hohenester, C. Sommer, Multiple interfacing between classical raytracing and waveoptical simulation approaches: a study on applicability and accuracy, Opt. Express 22 (13) (2014) 16048-16060, http://dx.doi.org/10.1364/0E.22. 016048.

[23] M.L. Griffith, J.W. Halloran, Scattering of ultraviolet radiation in turbid suspensions, J. Appl. Phys. 81 (6) (1997) 2538-2546, http://dx.doi.org/10.1063/ 1.364311.

[24] H. Horvath, Gustav Mie and the scattering and absorption of light by particles: Historic developments and basics, J. Quant. Spectrosc. Radiat. Transfer 110 (11) (2009) 787-799, http://dx.doi.org/10.1016/j.jqsit.2009.02.022.

[25] L. Demkowicz, Computing with Hp-Adaptive Finite Elements: Volume 1 - One and Two Dimensional Elliptic and Maxwell Problems, Chapman and Hall/CRC, 2006, p. 398.

[26] Comsol Multiphysics RF Module User 's Guide, Tech. rep., Comsol Multiphysics, 2014. URL www.comsol.com.

[27] J.D. Joannopoulos, S.G. Johnson, J.N. Winn, R.D. Meade, Photonic Crystals: Molding the Flow of Light, second ed, Princeton University Press, 2008, p. 286. URL http://ab-initio.mit.edu/book/.

[28] J.-M. Jin, The Finite Element Method in Electromagnetics, third ed, John Wiley \& Sons, Inc., Hoboken, New Jersey, 2014, p. 876. URL http://eu.wiley. com/WileyCDA/WileyTitle/productCd-1118571363.html.

[29] L. Demkowicz, L. Vardapetyan, Modeling of electromagnetic absorption / scattering problems using adaptive finite elements, Comput. Methods Appl. Mech. Engrg. 152 (1-2)(1998) 103-124, http://dx.doi.org/10.1016/s0045-7825(97)00184-9.

[30] J.-P. Berenger, A perfectly matched layer for the absorption of electromagnetic waves, J. Comput. Phys. 114 (2) (1994) 185-200, http://dx.doi.org/10. 1006/jcph.1994.1159.

[31] W. Shin, S. Fan, Choice of the perfectly matched layer boundary condition for frequency-domain Maxwell's equations solvers, J. Comput. Phys. 231 (8) (2012) 3406-3431, http://dx.doi.org/10.1016/j.jcp.2012.01.013.

[32] J.P. Webb, Application of the finite-element method to electromagnetic and electrical topics, Rep. Progr. Phys. 58 (12) (1995) 1673-1712, http: //dx.doi.org/10.1088/0034-4885/58/12/002.

[33] J.C. Nedelec, Mixed finite elements in R3, Numer. Math. 35 (3) (1980) 315-341, http://dx.doi.org/10.1007/BF01396415.

[34] P. Monk, Finite Element Methods for Maxwell's Equations, Oxford University Press, Newark, NJ, 2003, p. 464, http://dx.doi.org/10.1093/acprof: oso/9780198508885.001.0001.

[35] J. Pomplun, S. Burger, L. Zschiedrich, F. Schmidt, Adaptive finite element method for simulation of optical nano structures, Phys. Status Solidi (B) 244 (10) (2007) 3419-3434, http://dx.doi.org/10.1002/pssb.200743192.

[36] A. Navid, L. Pilon, Effect of polarization and morphology on the optical properties of absorbing nanoporous thin films, Thin Solid films 516 (12) (2008) 4159-4167, http://dx.doi.org/10.1016/j.tsf.2007.10.117.

[37] E. Andrzejewska, Photopolymerization kinetics of multifunctional monomers, Prog. Polym. Sci. 26 (4) (2001) 605-665, http://dx.doi.org/10.1016/ S0079-6700(01)00004-1.

[38] D.C. Watts, Reaction kinetics and mechanics in photo-polymerised networks, Dent. Mater. 21 (2005) 27-35, http://dx.doi.org/10.1016/j.dental.2004. 10.003. 
[39] G. Odian, Principles of Polymerization, John Wiley \& Sons, Inc., Hoboken, NJ, USA, 2004, http://dx.doi.org/10.1002/047147875X.

[40] A.S. Jariwala, F. Ding, A. Boddapati, V. Breedveld, M.A. Grover, C.L. Henderson, D.W. Rosen, Modeling effects of oxygen inhibition in mask-based stereolithography, Rapid Prototyping J. 17 (3) (2011) 168-175, http://dx.doi.org/10.1108/13552541111124734.

[41] H. Urbach, O. Janssen, S. van Haver, A. Wachters, On the modeling of optical systems containing elements of different scales, J. Modern Opt. 58 (5-6) (2011) 496-508, http://dx.doi.org/10.1080/09500340.2010.538736.

[42] A. Chatterjee, J.M. Jin, J.L. Volakis, Edge-based finite elements and vector ABC's applied to 3-D scattering, IEEE Trans. Antennas and Propagation 41 (2) (1993) 221-226, http://dx.doi.org/10.1109/8.214614.

[43] F. Aloui, L. Lecamp, P. Lebaudy, F. Burel, Relationships between refractive index change and light scattering during photopolymerization of acrylic composite formulations, J. Eur. Ceram. Soc. 36 (2016) 1805-1809, http://dx.doi.org/10.1016/j.jeurceramsoc.2016.01.033.

[44] E. Johansson, O. Lidström, J. Johansson, O. Lyckfeldt, E. Adolfsson, Influence of resin composition on the defect formation in alumina manufactured by stereolithography, Materials 10 (138)(2017) 11, http://dx.doi.org/10.3390/ma10020138.

[45] P.R. Cromwell, Polyhedra, Cambridge University Press, 1997, p. 451. 